\title{
Publisher Correction to: Deep learning- based image analysis methods for brightfield-acquired multiplex immunohistochemistry images
}

Danielle J. Fassler ${ }^{1 \dagger}$, Shahira Abousamra ${ }^{2 \dagger}$, Rajarsi Gupta ${ }^{3}$, Chao Chen ${ }^{3}$, Maozheng Zhao ${ }^{2}$, David Paredes ${ }^{2}$, Syeda Areeha Batool ${ }^{3}$, Beatrice S. Knudsen ${ }^{4}$, Luisa Escobar-Hoyos ${ }^{1,5}$, Kenneth R. Shroyer ${ }^{1}$, Dimitris Samaras ${ }^{2}$, Tahsin Kurc ${ }^{3}$ and Joel Saltz ${ }^{3^{*}}$

Publisher Correction to: Diagn Pathol 15, 100 (2020) https://doi.org/10.1186/s13000-020-01003-0

Following publication of the original article [1], the authors noticed that the figures were missing from the article. The accompanying figures are presented here. The publisher apologizes to the authors and readers for the inconvenience. The original article has been updated.

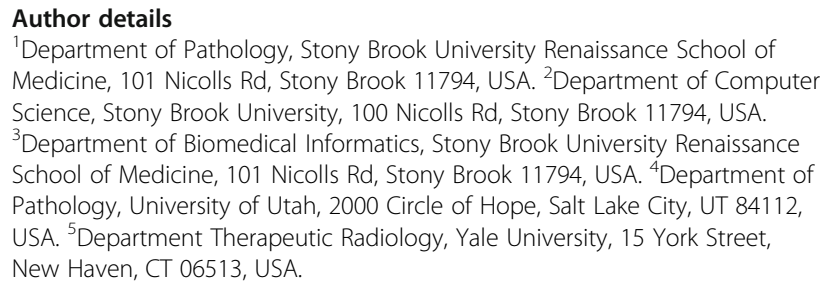

Published online: 24 September 2020

\section{Reference}

1. Fassler DJ, Abousamra S, Gupta R, et al. Deep learning-based image analysis methods for brightfield-acquired multiplex immunohistochemistry images. Diagn Pathol. 2020;15:100. https://doi.org/10.1186/s13000-020-01003-0.

* Correspondence: joel.saltz@stonybrookmedicine.edu

${ }^{\dagger}$ Danielle J. Fassler and Shahira Abousamra contributed equally to this work. ${ }^{3}$ Department of Biomedical Informatics, Stony Brook University Renaissance School of Medicine, 101 Nicolls Rd, Stony Brook 11794, USA

Full list of author information is available at the end of the article

Ready to submit your research? Choose BMC and benefit from:

- fast, convenient online submission

- thorough peer review by experienced researchers in your field

- rapid publication on acceptance

- support for research data, including large and complex data types

- gold Open Access which fosters wider collaboration and increased citations

- maximum visibility for your research: over 100M website views per year

At $\mathrm{BMC}$, research is always in progress.

Learn more biomedcentral.com/submissions

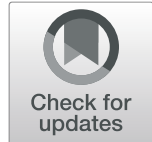

(c) The Author(s). 2020 Open Access This article is licensed under a Creative Commons Attribution 4.0 International License, which permits use, sharing, adaptation, distribution and reproduction in any medium or format, as long as you give appropriate credit to the original author(s) and the source, provide a link to the Creative Commons licence, and indicate if changes were made. The images or other third party material in this article are included in the article's Creative Commons. licence, unless indicated otherwise in a credit line to the material. If material is not included in the article's Creative Commons licence and your intended use is not permitted by statutory regulation or exceeds the permitted use, you will need to obtain permission directly from the copyright holder. To view a copy of this licence, visit http://creativecommons.org/licenses/by/4.0/ The Creative Commons Public Domain Dedication waiver (http://creativecommons.org/publicdomain/zero/1.0/) applies to the data made available in this article, unless otherwise stated in a credit line to the data. 


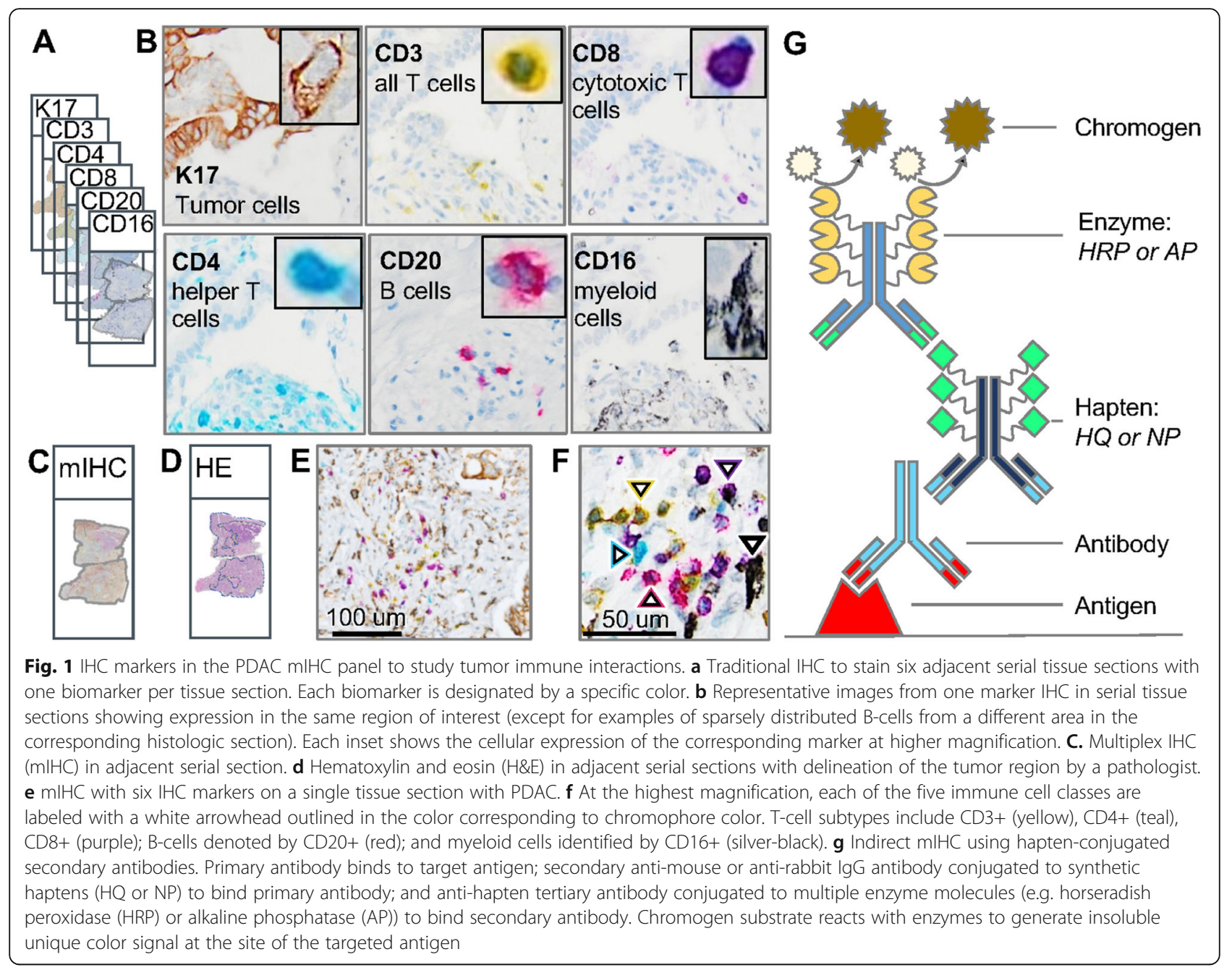



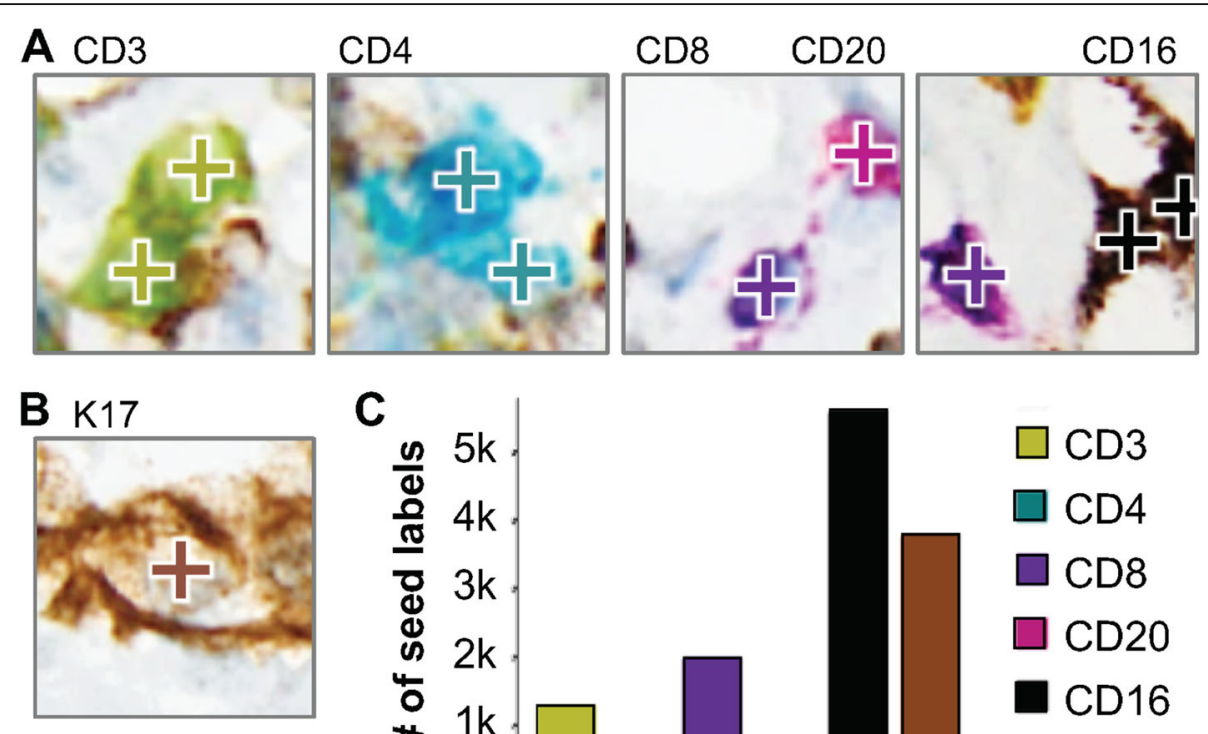

C
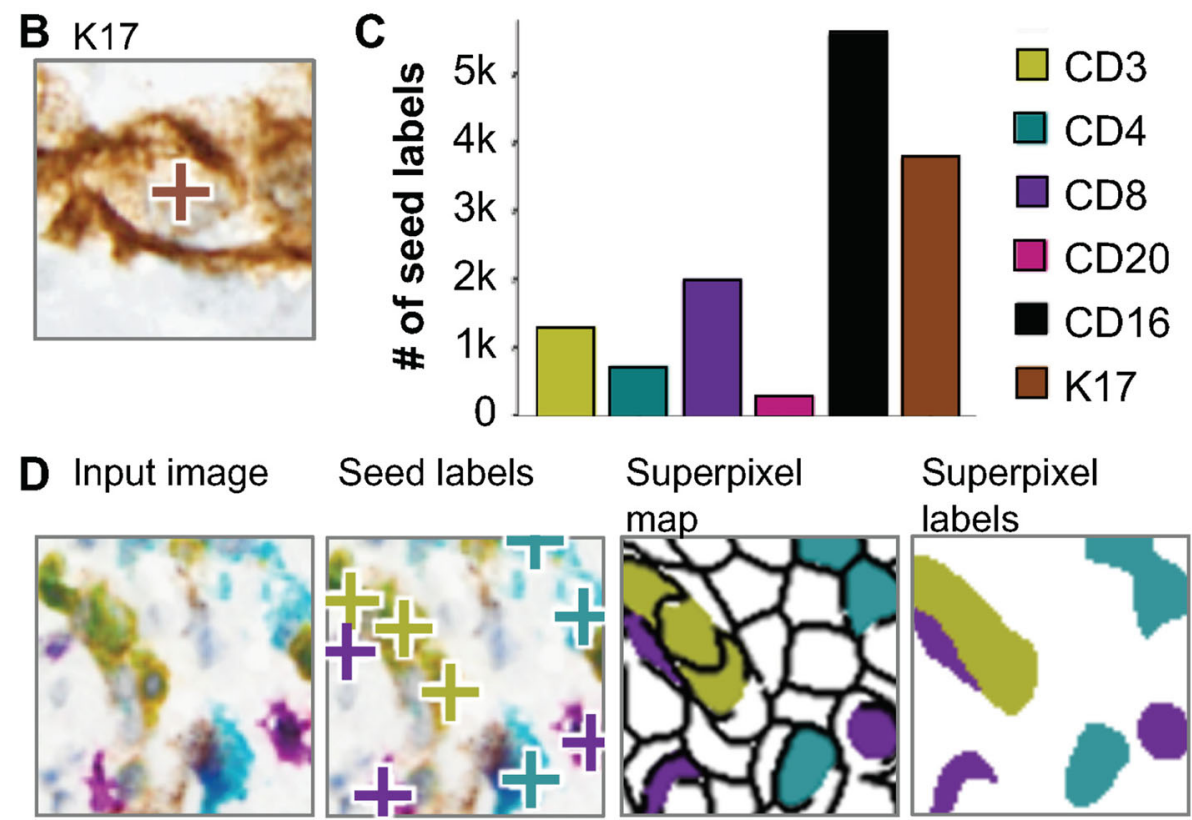

Superpixel

Superpixel

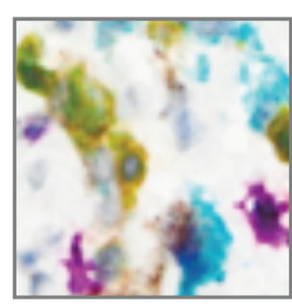

map labels

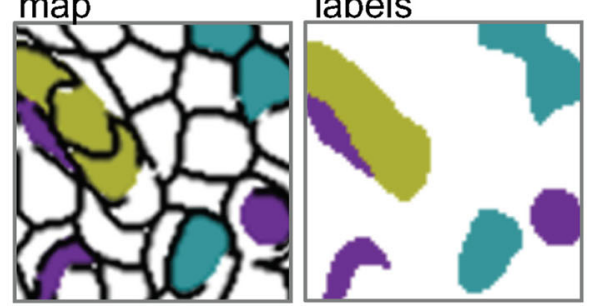

Fig. 2 Annotation of patches with seed labels and generation of per-pixel training data. a Examples of CD3+, CD4+, CD8+ and CD20+ lymphocytes, CD16+ myeloid cells and $\mathbf{b}$ K17+ PDAC tumor cells with seed labels overlaid (+). c Number of seed labels for each cell class, across all patches used for training. $\mathbf{d}$ Input image; input image with seed labels overlaid; superpixel map generated based on the input image, with superpixels containing different seed labels colored accordingly; and the superpixel labels used to train the models (based on seed labels and superpixel map) 


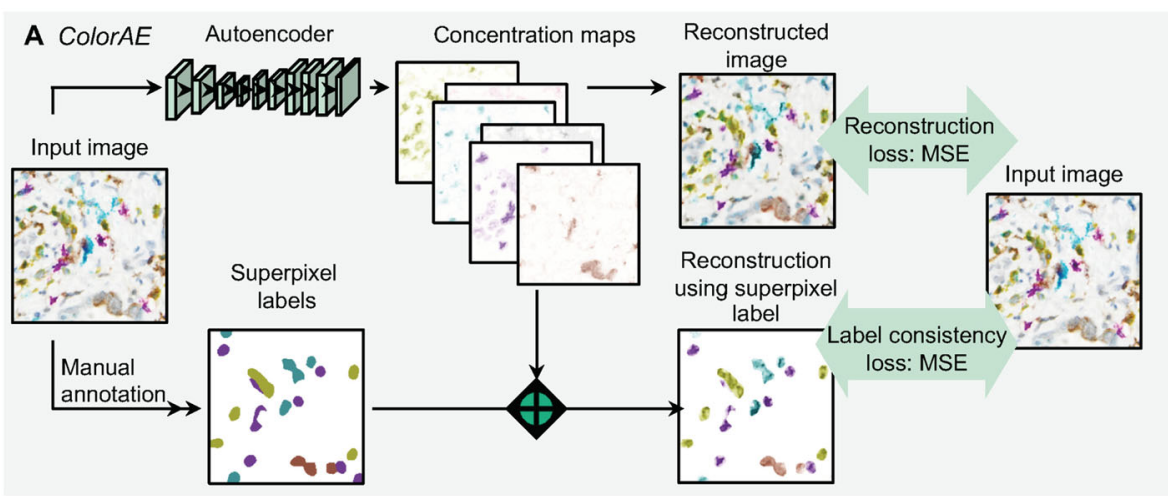

B U-Net
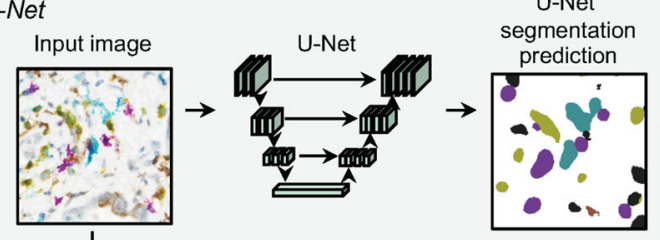

U-Ne

Manual annotations

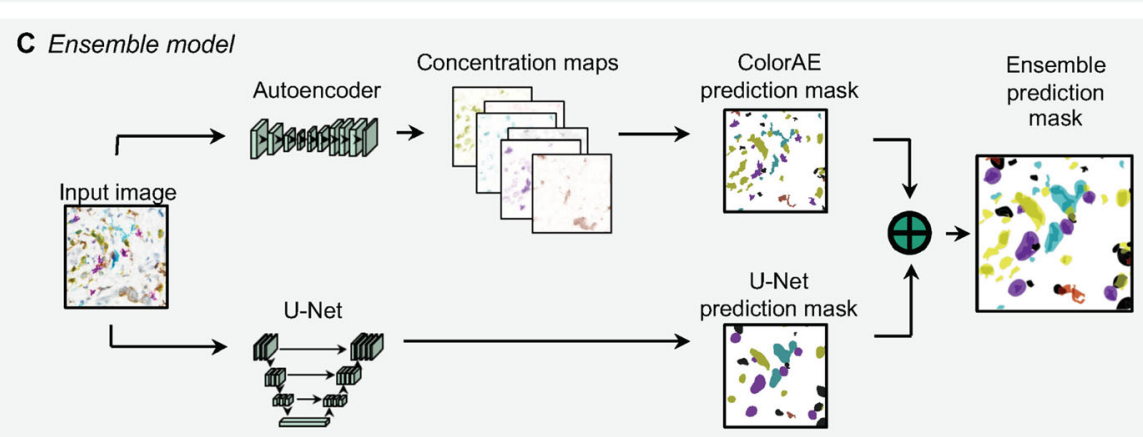

Fig. 3 Algorithm training. a ColorAE training. Input image is run through an autoencoder to yield concentration maps of each color (6 distinct mIHC stain colors: yellow, teal, purple, red, black, brown; blue hematoxylin nuclear counterstain; and background.) Two loss functions are applied to ensure that the reconstructed image has the highest fidelity to the original image and expert weak annotations. b U-Net training. Input image was run through a U-Net. Cross entropy loss function was applied to maximize fidelity to superpixel labels derived from manual annotation of the input image. c Ensemble method workflow. Input image is run through the autoencoder and U-Net to generate predictions as shown above 


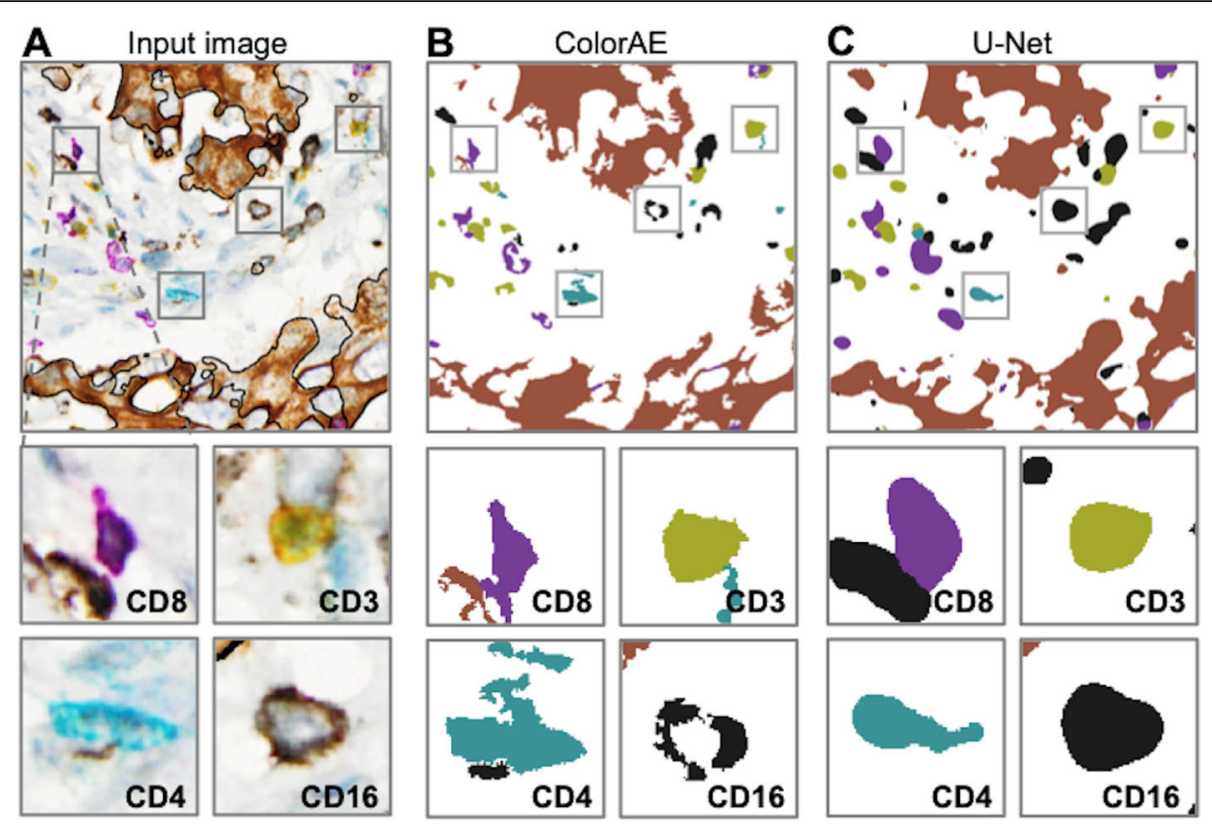

Fig. 4 ColorAE and U-Net predictions. a mIHC input image of tumor microenvironment, with a representative cell from each class of immune cells (magnified below). b-c ColorAE and U-Net prediction masks based on the original image 


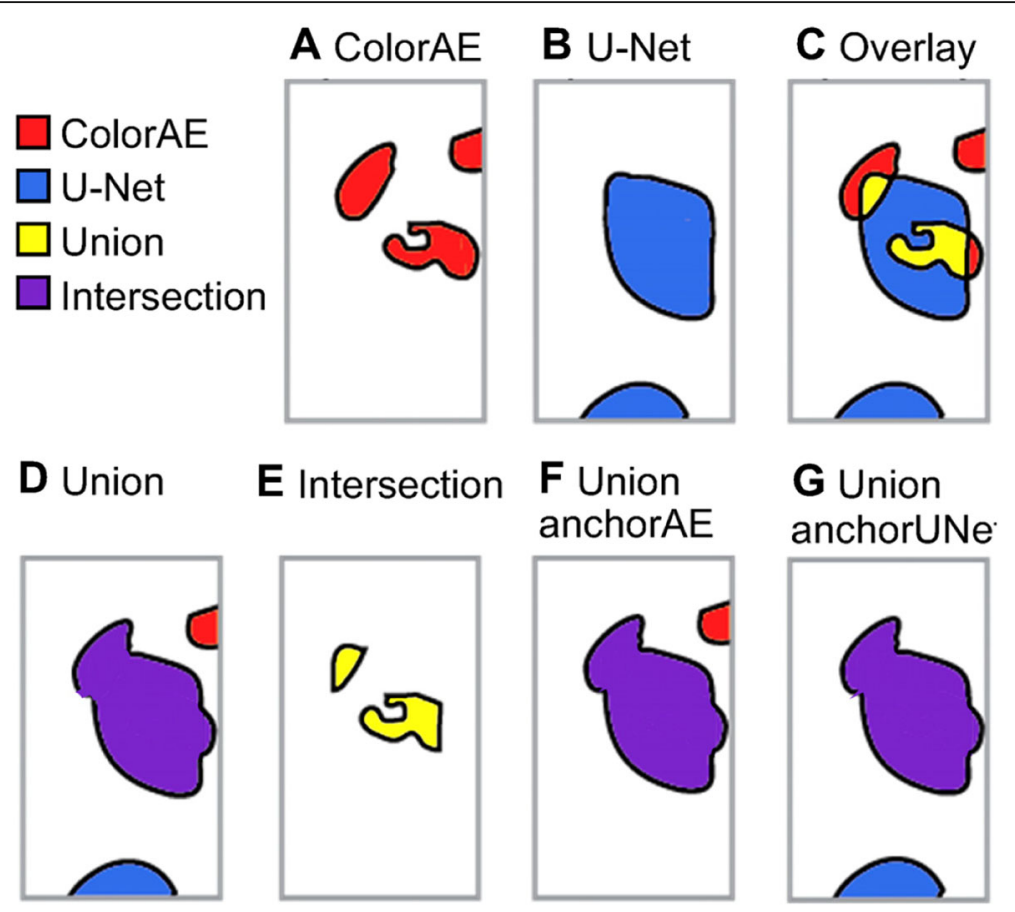

Fig. 5 ColorAE, U-Net, and ensemble predictions. All panels show predictions from the same cell class from the same input image. Top panel: a-c ColorAE and U-Net predictions are shown for a single cell class are shown individually and overlaid. Bottom panel, ensemble methods: $\mathbf{d}$ Union includes predictions from both ColorAE (red) and U-NET (blue): any overlapping predictions (detected by both algorithms) are merged into a single mask (purple). e Intersection includes the pixels detected by both algorithms, while excluding any areas of the mask detected by only one algorithm. f UnionanchorAE includes all masks detected by ColorAE and U-Net masks that intersect a ColorAE mask (union) while excluding UNet masks that do not intersect a ColorAE mask (only detected by the U-Net). $\mathbf{g}$ UnionanchorUNet includes all masks detected by U-Net and ColorAE masks that intersect a U-Net mask (union) while excluding ColorAE masks that do not intersect a U-Net mask (only detected by the ColorAE) 

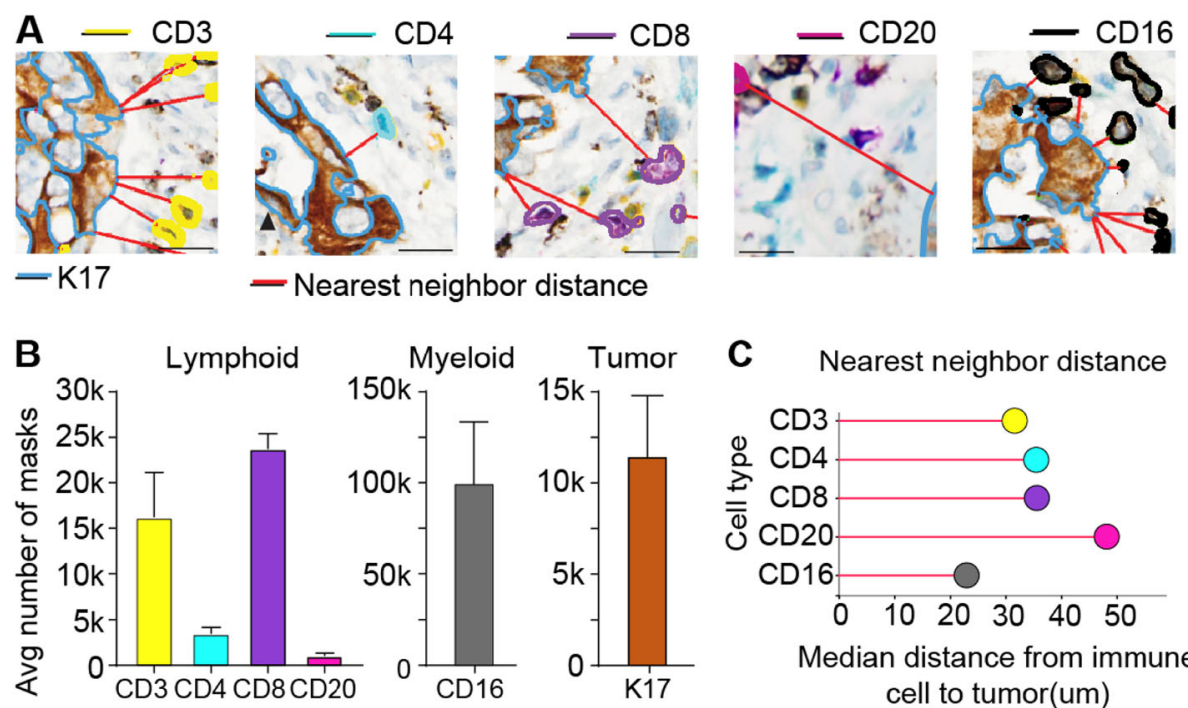

C Nearest neighbor distance

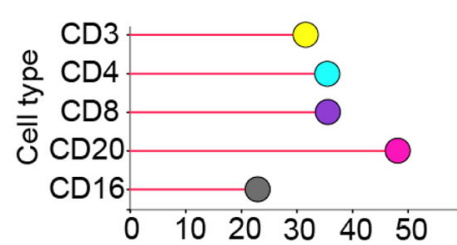

Median distance from immune cell to tumor(um)

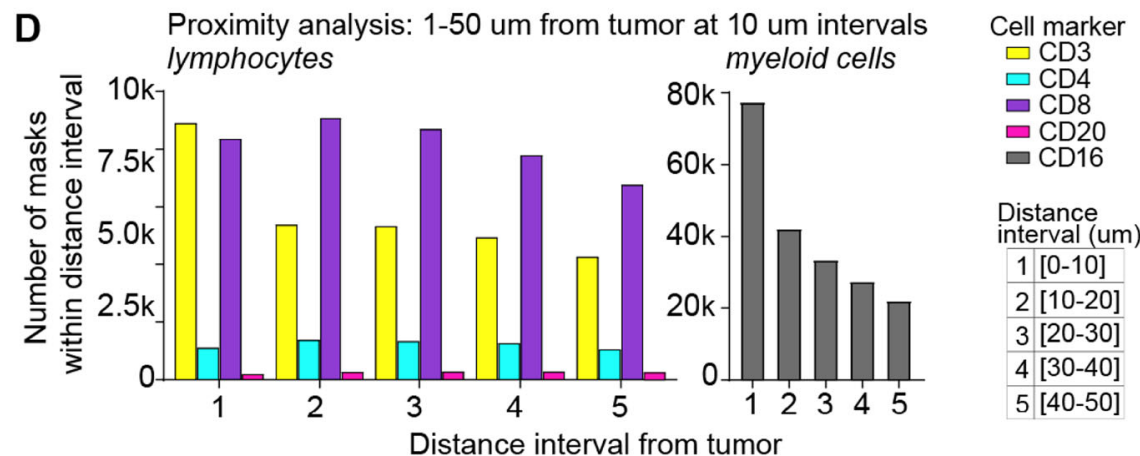

Fig. 6 Example of analyses describing immune-tumor spatial relationships in mIHC-stained PDAC tissue. a Representative images of segmentation boundaries of detected tumor nests and immune cells labelled with IHC biomarkers: K17 (blue segmentation boundary), CD3 (yellow boundary), CD4 (teal boundary), CD8 (purple boundary), CD20 (magenta boundary), and CD16 (black boundary). Red lines indicate nearest neighbor distance vector connecting each immune cell to the nearest tumor nest. $\mathbf{b}$ Average number of masks per case for each of the different cell classes in three WSIs. c Median nearest neighbor distances for each immune cell class across cases. d Proximity analysis showing the number of detected masks for each cell class at 10 um distance intervals from the tumor boundary. *Note: Nearest Neighbor analyses are asymmetric and Nearest Neighbor analyses from each tumor nest to nearest immune cell are shown in supplemental Fig. 4 